\title{
On the relation between Schmidt coefficients and entanglement
}

\author{
Paolo Aniello \\ Dipartimento di Scienze Fisiche dell'Università di Napoli "Federico II" \& INFN - Sezione di Napoli \& \\ Facoltà di Scienze Biotecnologiche, Università di Napoli "Federico II", via Cintia I-80126 Napoli, Italy \\ Cosmo Lupo \\ Dipartimento di Fisica, Università di Camerino, I-62032 Camerino, Italy
}

September 10, 2018

\begin{abstract}
We consider the Schmidt decomposition of a bipartite density operator induced by the Hilbert-Schmidt scalar product, and we study the relation between the Schmidt coefficients and entanglement. First, we define the Schmidt equivalence classes of bipartite states. Each class consists of all the density operators (in a given bipartite Hilbert space) sharing the same set of Schmidt coefficients. Next, we review the role played by the Schmidt coefficients with respect to the separability criterion known as the 'realignment' or 'computable cross norm' criterion; in particular, we highlight the fact that this criterion relies only on the Schmidt equivalence class of a state. Then, the relevance - with regard to the characterization of entanglement - of the "symmetric polynomials' in the Schmidt coefficients and a new family of separability criteria that generalize the realignment criterion are discussed. Various interesting open problems are proposed.
\end{abstract}

\section{Introduction}

The study of the structure and of the properties of the convex body of states of a quantum system is still nowadays a source of intriguing problems and an active area of research, especially in the case of quantum systems with a finite-dimensional Hilbert space [1]. A great impulse to this area of research has been impressed by the new applications related to quantum information science [2]. In particular, the interest of many scientists is focused on the study of bipartite (and multipartite) quantum systems, in which the typical quantum phenomenon known as entanglement can be present and has remarkable consequences. Let us recall [3] that a state $\rho$ of a bipartite quantum system is said to be separable if it can be written as a convex sum of product states

$$
\rho=\sum_{k} p_{k} \rho_{k}^{\mathrm{A}} \otimes \rho_{k}^{\mathrm{B}}, \quad p_{k}>0, \quad \sum_{k} p_{k}=1
$$

otherwise, it is said to be entangled. Clearly, an important and difficult task is to determine whether a certain quantum state is separable or not. To accomplish this task several necessary and/or sufficient criteria have been formulated and discussed in literature; however, no universal - i.e. holding for any dimension of the local Hilbert spaces - necessary-sufficient criterion that is practically implementable is known so far [1, 4].

In the following, we will consider the case of a bipartite quantum system with a finite-dimensional carrier Hilbert space $\mathcal{H}=\mathcal{H}_{\mathrm{A}} \otimes \mathcal{H}_{\mathrm{B}}$, with $\mathcal{H}_{\mathrm{A}} \cong \mathbb{C}^{N_{\mathrm{A}}}$ and $\mathcal{H}_{\mathrm{B}} \cong \mathbb{C}^{N_{\mathrm{B}}}, N_{\mathrm{A}}, N_{\mathrm{B}} \geq 2$. Observe that the states of the local 
subsystems $\mathrm{A}$ and $\mathrm{B}$ are characterized, respectively, by $N_{\mathrm{A}}^{2}-1$ and $N_{\mathrm{B}}^{2}-1$ real parameters (being unit-trace positive selfadjoint operators), while the states of the global system are characterized by $N_{\mathrm{A}}^{2} N_{\mathrm{B}}^{2}-1$ real parameters; this fact is at the very root of the quantum entanglement. It is then not surprising that practically implementable separability criteria can be obtained by considering a convenient parametrization of the set of states of the composite system.

Consider, for instance, the Fano parametrization [5] of a $2 \otimes 2$ bipartite state - i.e.

$$
\rho=\frac{1}{4}\left(\mathbb{I}_{2}^{\mathrm{A}} \otimes \mathbb{I}_{2}^{\mathrm{B}}+x_{a} \sigma_{a}^{\mathrm{A}} \otimes \mathbb{I}_{2}^{\mathrm{B}}+y_{\alpha} \mathbb{I}_{2}^{\mathrm{A}} \otimes \sigma_{\alpha}^{\mathrm{B}}+\xi_{a \alpha} \sigma_{a}^{\mathrm{A}} \otimes \sigma_{\alpha}^{\mathrm{B}}\right) .
$$

(sum over the repeated indices being understood), where $\left\{\sigma_{a}\right\}_{a=1}^{3}$ are the Pauli matrices — or its $N_{\mathrm{A}} \otimes N_{\mathrm{B}}$ generalization in terms of the (traceless selfadjoint) 'Gell-Mann matrices' $\left\{\Lambda_{a}^{\mathrm{A}} \mid a=1, \ldots, N_{\mathrm{A}}^{2}-1\right\}$ and $\left\{\Lambda_{\alpha}^{\mathrm{B}} \mid \alpha=1, \ldots, N_{\mathrm{B}}^{2}-1\right\}$ :

$$
\rho=\frac{1}{N_{\mathrm{A}} N_{\mathrm{B}}}\left(\mathbb{I}_{N_{\mathrm{A}}}^{\mathrm{A}} \otimes \mathbb{I}_{N_{\mathrm{B}}}^{\mathrm{B}}+x_{a} \Lambda_{a}^{\mathrm{A}} \otimes \mathbb{I}_{N_{\mathrm{B}}}^{\mathrm{B}}+y_{\alpha} \mathbb{I}_{N_{\mathrm{A}}}^{\mathrm{A}} \otimes \Lambda_{\alpha}^{\mathrm{B}}+\xi_{a \alpha} \Lambda_{a}^{\mathrm{A}} \otimes \Lambda_{\alpha}^{\mathrm{B}}\right)
$$

The coefficients $\left\{x_{a}\right\},\left\{y_{\alpha}\right\},\left\{\xi_{a \alpha}\right\}$ - that are subjected to suitable constraints which guarantee the positivity of the density matrix [6] (while the normalization of the trace is ensured by the form of the parametrization itself) turn out to be relevant for characterizing the entanglement of the state $\rho$. In particular, the matrix formed by the coefficients $\left\{\xi_{a \alpha}\right\}$ has been considered for obtaining new separability criteria, see [7].

A natural generalization of decomposition (3) is achieved by considering 'local bases of observables' (or 'local orthogonal observables' [8])

$$
\left\{F_{a}^{\mathrm{A}}: \mathcal{H}_{\mathrm{A}} \rightarrow \mathcal{H}_{\mathrm{A}} \mid a=1, \ldots, N_{\mathrm{A}}^{2}\right\}, \quad\left\{F_{\alpha}^{\mathrm{B}}: \mathcal{H}_{\mathrm{B}} \rightarrow \mathcal{H}_{\mathrm{B}} \mid \alpha=1, \ldots, N_{\mathrm{B}}^{2}\right\}
$$

in the 'local Hilbert-Schmidt spaces', i.e. orthonormal bases in the real Hilbert spaces of selfadjoint linear operators in $\mathcal{H}_{\mathrm{A}}, \mathcal{H}_{\mathrm{B}}$ (endowed with the real Hilbert-Schmidt scalar product):

$$
\begin{aligned}
& \left\langle F_{a}^{\mathrm{A}}, F_{b}^{\mathrm{A}}\right\rangle_{\mathrm{HS}}=\operatorname{tr}\left(F_{a}^{\mathrm{A}} F_{b}^{\mathrm{A}}\right)=\delta_{a b}, \\
& \left\langle F_{\alpha}^{\mathrm{B}}, F_{\beta}^{\mathrm{B}}\right\rangle_{\mathrm{HS}}=\operatorname{tr}\left(F_{\alpha}^{\mathrm{B}} F_{\beta}^{\mathrm{B}}\right)=\delta_{\alpha \beta} .
\end{aligned}
$$

Generalizing the expression (3), one can now write

$$
\rho=\tilde{\rho}_{a \alpha} F_{a}^{\mathrm{A}} \otimes F_{\alpha}^{\mathrm{B}},
$$

where:

$$
\tilde{\rho}_{a \alpha}=\left\langle F_{a}^{\mathrm{A}} \otimes F_{\alpha}^{\mathrm{B}}, \rho\right\rangle_{\mathrm{HS}}=\operatorname{tr}\left(F_{a}^{\mathrm{A}} \otimes F_{\alpha}^{\mathrm{B}} \rho\right) .
$$

Note that the $N_{\mathrm{A}}^{2} \times N_{\mathrm{B}}^{2}$ matrix of coefficients $\tilde{\rho} \equiv\left[\tilde{\rho}_{a \alpha}\right]$ is formed by — at least in principle — measurable quantities; namely, the expectation values of (local) quantum observables.

Even more generally, one can relax the assumption that the local bases (4) consist of observables; i.e. one can assume that they are generic orthonormal bases in the complex vector spaces of linear operators - in $\mathcal{H}_{\mathrm{A}}$ and $\mathcal{H}_{\mathrm{B}}$, respectively - endowed with the complex Hilbert-Schmidt scalar product.

The matrix of coefficients $\tilde{\rho}$ has recently been considered in connection with the separability problem, see refs. [9, 10]. In particular, it turns out that the singular values [11] of this matrix coincide with the (mixed state) Schmidt coefficients of $\rho$ (see below). Moreover, the sum of these (non-negative) coefficients is equal to the computable cross norm of the state $\rho$, a quantity which is at the base of the remarkable separability criterion known as the 'realignment' or 'computable cross norm' criterion [12, 13, 14]. 
The aim of the present contribution is to investigate the role played by the Schmidt coefficients in the characterization of the bipartite entanglement. There is a very sound reason for being interested in such a question: the Schmidt coefficients have a transparent mathematical meaning and are easily computable from physically measurable quantities (actually we will see that they are, in principle, physically measurable quantities themselves). We will also indicate some interesting open problems.

The paper is organized as follows. In Sect. 2] we recall the notions of 'Schmidt decomposition', of 'Schmidt coefficients' and of 'Schmidt equivalence class' of bipartite states. In Sects. 3 and 4 we will try to highlight the relation between entanglement and the Schmidt coefficients. In particular, in Sect. 3, we will consider the link between entanglement and the sum of the Schmidt coefficients of a bipartite state; while, in Sect. 4 we will argue that a stricter characterization of entanglement can be achieved by considering the whole set of the 'symmetric polynomials' in the Schmidt coefficients rather than the sum of the Schmidt coefficients alone (which is the symmetric polynomial of degree one). The results outlined in Sect. 4, by virtue of the well known relation between quantum states and quantum maps, allow to to obtain — see Sect. 5 — an interesting characterization of entanglement breaking quantum channels. In Sect. 6, a recently discovered new class of separability criteria see [9] — is described. This class — that contains as particular cases various separability criteria already present in the literature, including the realignment criterion — relies on the sum of the Schmidt coefficients of a 'transformed operator' suitably associated with the bipartite state whose separability is under scrutiny. Eventually, in Sect. 7 a few conclusion are drawn.

\section{Schmidt coefficients and Schmidt equivalence classes of bipartite states}

The Schmidt decomposition plays a central role in the characterization of pure state entanglement in the bipartite setting [15]. Let us recall (see [16]) that any state vector $|\psi\rangle$, belonging to the bipartite Hilbert space $\mathcal{H}=\mathcal{H}_{\mathrm{A}} \otimes \mathcal{H}_{\mathrm{B}}$, can always be decomposed as

$$
|\psi\rangle=\sum_{k=1}^{\delta} \lambda_{k}\left|\psi_{k}^{\mathrm{A}}\right\rangle \otimes\left|\psi_{k}^{\mathrm{B}}\right\rangle, \quad \lambda_{k} \geq 0,
$$

where the vectors in the decomposition are tensor products of local orthonormal systems $-\left\langle\psi_{h}^{\mathrm{A}} \mid \psi_{k}^{\mathrm{A}}\right\rangle=\left\langle\psi_{h}^{\mathrm{B}} \mid \psi_{k}^{\mathrm{B}}\right\rangle=$ $\delta_{h k}-$ and $\delta \equiv \min \left\{N_{\mathrm{A}}, N_{\mathrm{B}}\right\}$. This expression, called 'the Schmidt decomposition of the state vector $|\psi\rangle$ ' (representative of a pure state), is non-unique, while the Schmidt coefficients $\left\{\lambda_{k}\right\}_{k=1}^{\delta}$ are uniquely determined (up to re-ordering). The set of the Schmidt coefficients associated with a pure state completely characterizes the separability of this state; accordingly, any measure of entanglement on pure states can be written as a function of the Schmidt coefficients [15]. Unfortunately, this remarkable property is lost in the more general context of mixed states. In order to clarify this point, one has to extend the notion of Schmidt coefficients to bipartite mixed states. We will follow the approach of refs. [9, 10].

Let us consider, then, the convex body $\mathcal{D}(\mathcal{H})$ of states of a quantum system 11 with carrier Hilbert space $\mathcal{H}=$ $\mathcal{H}_{\mathrm{A}} \otimes \mathcal{H}_{\mathrm{B}}$. The set $\mathcal{D}(\mathcal{H})$ can be regarded as a convex subset of the complex vector space $\mathcal{L}(\mathcal{H})$ of linear operators in $\mathcal{H}$. The vector space $\mathcal{L}(\mathcal{H})$ becomes a Hilbert space if endowed with the (complex) Hilbert-Schmidt scalar product:

$$
\langle A, B\rangle_{\mathrm{HS}}:=\operatorname{tr}\left(A^{\dagger} B\right), \quad A, B \in \mathcal{L}(\mathcal{H}) .
$$

\footnotetext{
${ }^{1} \operatorname{Since} \operatorname{dim}(\mathcal{H})<\infty$, by Gleason's theorem we can identify $\mathcal{D}(\mathcal{H})$ with the set of density operators (unit trace positive linear operators) in $\mathcal{H}$.
} 
We will denote by $\|\cdot\|_{\mathrm{HS}}$ the associated norm:

$$
\|A\|_{\mathrm{HS}}:=\sqrt{\operatorname{tr}\left(A^{\dagger} A\right)} .
$$

Hence, given a density operator $\rho \in \mathcal{D}\left(\mathcal{H}_{\mathrm{A}} \otimes \mathcal{H}_{\mathrm{B}}\right) \subset \mathcal{L}(\mathcal{H})=\mathcal{L}\left(\mathcal{H}_{\mathrm{A}}\right) \otimes \mathcal{L}\left(\mathcal{H}_{\mathrm{B}}\right)$, there always exist local orthonormal systems $\left\{E_{a}^{\mathrm{A}}\right\}_{a=1}^{\mathrm{d}},\left\{E_{b}^{\mathrm{B}}\right\}_{b=1}^{\mathrm{d}}-\mathrm{d} \equiv \min \left\{N_{\mathrm{A}}^{2}, N_{\mathrm{B}}^{2}\right\}=\delta^{2}-$ such that the density matrix $\rho$ admits a decomposition of the form

$$
\rho=\sum_{a=1}^{\mathrm{d}} \mu_{a} E_{a}^{\mathrm{A}} \otimes E_{a}^{\mathrm{B}},
$$

where the coefficients $\left\{\mu_{a}\right\}_{a=1}^{d}$ are non-negative. We stress that the the set of coefficients $\left\{\mu_{a}\right\}_{a=1}^{d}$ is uniquely determined, and these coefficients are the (mixed state) Schmidt coefficients of the density operator $\rho$. In the case of a pure state $\rho=|\psi\rangle\langle\psi|$ (now realized as a 1-dimensional projector), they are related in a simple way to the state vector Schmidt coefficients of decomposition (9), i.e. $\left\{\mu_{a}\right\}_{a=1}^{\mathrm{d}}=\left\{\lambda_{h} \lambda_{k}\right\}_{h, k=1}^{\delta}$. One can easily check that a state $\rho \in \mathcal{D}(\mathcal{H})$ is a separable pure state $-\rho=\left|\psi^{\mathrm{A}}\right\rangle\left\langle\psi^{\mathrm{A}}|\otimes| \psi^{\mathrm{B}}\right\rangle\left\langle\psi^{\mathrm{B}}\right|-$ if and only if the set of its Schmidt coefficients is of the form $\left\{\mu_{a}\right\}_{a=1}^{d}=\{1,0,0, \ldots\}$.

Remark 2.1 It is clear that a Schmidt decomposition of the form (12) can actually be written for any operator belonging to $\mathcal{L}(\mathcal{H})$.

Note that in decomposition (12) (like in (9)) we allow the possibility that some Schmidt coefficients are zero, while, in the literature, usually only non-zero Schmidt coefficients are taken into account. Hence, according to our convention, in general the set of the Schmidt coefficients $\left\{\mu_{a}\right\}_{a=1}^{d}$ may contain both zeroes and repeated elements. The number of nonzero coefficients in the set $\left\{\mu_{a}\right\}_{a=1}^{\mathrm{d}}$ is called the Schmidt rank of $\rho$, and will be denoted by $\mathrm{R}$. Usually, the Schmidt coefficients are arranged in non-increasing order, but any particular ordering is irrelevant for our purposes.

It is easy to verify that the Schmidt coefficients of $\rho \in \mathcal{D}(\mathcal{H})$ coincide with the singular values of the matrix $\tilde{\rho}$ — see (8) — for any couple of local orthonormal bases in $\mathcal{L}\left(\mathcal{H}_{\mathrm{A}}\right)$ and $\mathcal{L}\left(\mathcal{H}_{\mathrm{B}}\right)$. Since we can regard $\mathcal{D}(\mathcal{H})$ also as a convex subset of the real Hilbert space $\mathcal{L}_{\mathbb{R}}(\mathcal{H})=\mathcal{L}_{\mathbb{R}}\left(\mathcal{H}_{\mathrm{A}}\right) \otimes \mathcal{L}_{\mathbb{R}}\left(\mathcal{H}_{\mathrm{B}}\right)$ of selfadjoint linear operators in $\mathcal{H}$ (endowed with the real Hilbert-Schmidt scalar product), then there always exists a Schmidt decomposition of $\rho$ of the form (12) where the orthonormal systems $\left\{E_{a}^{\mathrm{A}}\right\}_{a=1}^{\mathrm{d}}$ and $\left\{E_{b}^{\mathrm{B}}\right\}_{b=1}^{\mathrm{d}}$ consist of observables. Hence, not only the Schmidt coefficients are easily computable from physically measurable quantities (the entries of the matrix $\tilde{\rho}$, associated with given local bases of observables), but they are physically measurable quantities themselves:2

$$
\mu_{a}=\operatorname{tr}\left(E_{a}^{\mathrm{A}} \otimes E_{a}^{\mathrm{B}} \rho\right) .
$$

We have already mentioned the fact that the set of the Schmidt coefficients of a bipartite state is uniquely determined by this state; on the other hand, one can show that a set of Schmidt coefficients does not identify a unique quantum state (or, more generally, a unique linear operator). As it turns out that there is a relation between the Schmidt coefficients and entanglement [9, 10], it is worth wondering to what extent these coefficients alone are able to witness the presence of entanglement and, in this spirit, introducing a suitable 'Schmidt equivalence relation' (see [10]).

\footnotetext{
${ }^{2}$ Note, however, that the observables $\left\{E_{a}^{\mathrm{A}}\right\}_{a=1}^{\mathrm{d}}$ and $\left\{E_{b}^{\mathrm{B}}\right\}_{b=1}^{\mathrm{d}}$ will depend on the state $\rho$.
} 
Definition 2.2 We say that two bipartite density operators belonging to $\mathcal{D}(\mathcal{H})$ are Schmidt equivalent if they share the same set of Schmidt coefficients.

Observe that for any $\rho \in \mathcal{D}(\mathcal{H})-$ since $\sum_{a=1}^{\mathrm{d}} \mu_{a}^{2}=\langle\rho, \rho\rangle_{\mathrm{HS}}=\operatorname{tr}\left(\rho^{2}\right)$ - we have:

$$
\sum_{a=1}^{\mathrm{d}} \mu_{a}^{2}=1 \Leftrightarrow \rho \text { pure state; } \quad \sum_{a=1}^{\mathrm{d}} \mu_{a}^{2}<1 \quad \Leftrightarrow \quad \rho \text { non-pure state. }
$$

Therefore, the Schmidt equivalence relation induces in $\mathcal{D}(\mathcal{H})$ two kinds of equivalence classes: the pure state Schmidt equivalence classes (composed by pure states only) and the non-pure state Schmidt equivalence classes. The first kind of equivalence classes can be easily characterized [17], while a 'complete' characterization of the second kind of classes seems to be a difficult problem.

The pure state Schmidt equivalence classes are known to be smooth manifolds [17]. Whether this result can be extended to all the Schmidt equivalence classes of bipartite states is an interesting open problem. The technical difficulty in solving this problem consists in the difficulty of verifying whether there exist Lie groups acting smoothly and transitively on each Schmidt equivalence class, see [10]. In any case, it would be interesting to achieve a more complete understanding of the structure and the properties of the Schmidt equivalence classes of bipartite states.

\section{Entanglement and the sum of the Schmidt coefficients}

In this section, we will consider the role played by the Schmidt coefficients in the detection of entanglement. We will first focus our attention on the so-called 'computable cross norm' (CCN), and then we will discuss its relation with the 'realignment criterion' (RC) for separability. Our exposition will be rather concise and will not include proofs; the reader interested in the details may consult refs. [9, 12, 13, 14].

The $\mathrm{CCN}$ on $\mathcal{L}(\mathcal{H})=\mathcal{L}\left(\mathcal{H}_{\mathrm{A}}\right) \otimes \mathcal{L}\left(\mathcal{H}_{\mathrm{B}}\right)$ is a norm $\|\cdot\|_{\mathrm{CCN}}: \mathcal{L}(\mathcal{H}) \rightarrow \mathbb{R}^{+}$defined as follows:

$$
\begin{aligned}
\|C\|_{\mathrm{CCN}}:= & \inf \left\{\sum_{k=1}^{n}\left\|A_{k}\right\|_{\mathrm{HS}}\left\|B_{k}\right\|_{\mathrm{HS}}: C=\sum_{k=1}^{n} A_{k} \otimes B_{k},\right. \\
& \text { with } \left.\left\{A_{k}\right\}_{k=1}^{n} \subset \mathcal{L}\left(\mathcal{H}_{\mathrm{A}}\right),\left\{B_{k}\right\}_{k=1}^{n} \subset \mathcal{L}\left(\mathcal{H}_{\mathrm{B}}\right), n \in \mathbb{N}\right\},
\end{aligned}
$$

for any $C \in \mathcal{L}(\mathcal{H})$. It is possible to show that it is a cross norm on $\mathcal{L}(\mathcal{H})$, namely,

$$
\|A \otimes B\|_{\mathrm{CCN}}=\|A\|_{\mathrm{HS}}\|B\|_{\mathrm{HS}}, \quad \forall A \in \mathcal{L}\left(\mathcal{H}_{\mathrm{A}}\right), \forall B \in \mathcal{L}\left(\mathcal{H}_{\mathrm{B}}\right) .
$$

What is the relation between the $\mathrm{CCN}$ and the Schmidt coefficients is not immediately evident. However, it is possible to prove the following fact. Let $C$ be an operator in $\mathcal{L}(\mathcal{H})$ - in particular, a bipartite state $\rho \equiv C \in \mathcal{D}(\mathcal{H})$ — and let $\left\{\mu_{a}\right\}_{a=1}^{\mathrm{d}}$ be the set of its Schmidt coefficients; then, the following relation holds:

$$
\|C\|_{\mathrm{CCN}}=\sum_{a=1}^{\mathrm{d}} \mu_{a}
$$

But, as already observed, the Schmidt coefficients of $C$ coincide with the singular values of the matrix of coefficients of the decomposition of $C$ with respect to any product orthonormal basis in the tensor product Hilbert space 
$\mathcal{L}(\mathcal{H})=\mathcal{L}\left(\mathcal{H}_{\mathrm{A}}\right) \otimes \mathcal{L}\left(\mathcal{H}_{\mathrm{B}}\right)$. Hence, the $\mathrm{CCN}$ is easily computable, computable cross norm indeed! Explicitly, it turns out that the sum of the Schmidt coefficients of $\rho$ coincides with the trace norm of the matrix $\tilde{\rho}-$ see (8) for any choice of the local bases $\left\{F_{a}^{\mathrm{A}} \mid a=1, \ldots, N_{\mathrm{A}}^{2}\right\},\left\{F_{\alpha}^{\mathrm{B}} \mid \alpha=1, \ldots, N_{\mathrm{B}}^{2}\right\}$ in $\mathcal{L}\left(\mathcal{H}_{\mathrm{A}}\right)$ and $\mathcal{L}\left(\mathcal{H}_{\mathrm{B}}\right)$, respectively (here we are not assuming that these orthonormal bases consist of observables):

$$
\|\rho\|_{\mathrm{CCN}}=\sum_{a=1}^{\mathrm{d}} \mu_{a}=\|\tilde{\rho}\|_{\mathrm{tr}}
$$

where

$$
\|\tilde{\rho}\|_{\operatorname{tr}}:=\operatorname{tr}\left(\sqrt{\tilde{\rho}^{\dagger} \tilde{\rho}}\right)
$$

We now come to the RC. This criterion admits two formulations. According to its more popular formulation [12] — which explains the term 'realignment criterion' — this separability criterion is stated as follows (we will be rather sketchy; for the details, see [9]). Let $\rho \in \mathcal{D}(\mathcal{H})$ be a bipartite state and let $\check{\rho}$ be the $N_{\mathrm{A}} N_{\mathrm{B}} \times N_{\mathrm{A}} N_{\mathrm{B}}$ representative matrix of this operator with respect to any product orthonormal basis in the Hilbert space $\mathcal{H}=\mathcal{H}_{\mathrm{A}} \otimes \mathcal{H}_{\mathrm{B}}$. One can associate with the matrix $\check{\rho}$ a realigned matrix $\check{\rho}^{\mathrm{R}}$ (which is a $N_{\mathrm{A}}^{2} \times N_{\mathrm{B}}^{2}$ matrix obtained from $\check{\rho}$ via a 'reshuffling' operation, a suitable re-arrangement of the entries of $\check{\rho}$ ), and it turns out that the following implication holds:

$$
\rho \text { separable } \Rightarrow\left\|\check{\rho}^{\mathrm{R}}\right\|_{\text {tr }} \leq 1 .
$$

One can show that the realigned matrix $\check{\rho}^{\mathrm{R}}$ coincides with the matrix $\tilde{\rho}$ for a suitable choice of the local bases $\left\{F_{a}^{\mathrm{A}} \mid a=1, \ldots, N_{\mathrm{A}}^{2}\right\},\left\{F_{\alpha}^{\mathrm{B}} \mid \alpha=1, \ldots, N_{\mathrm{B}}^{2}\right\}$ in $\mathcal{L}\left(\mathcal{H}_{\mathrm{A}}\right), \mathcal{L}\left(\mathcal{H}_{\mathrm{B}}\right)$. It follows that

$$
\|\rho\|_{\mathrm{CCN}}=\left\|\check{\rho}^{\mathrm{R}}\right\|_{\mathrm{tr}}
$$

Therefore, the RC admits the following remarkable formulation involving the sum of the Schmidt coefficients:

$$
\rho \text { separable } \Rightarrow\|\rho\|_{\mathrm{CCN}}=\sum_{a=1}^{\mathrm{d}} \mu_{a} \leq 1 .
$$

This second more conceptual formulation of the RC explains the alternative term 'computable cross norm criterion'. This formulation also highlights the fact that the RC relies only on the Schmidt equivalence class of a bipartite state.

\section{Entanglement and the symmetric polynomials in the Schmidt coefficients}

The RC is based on the evaluation of a single quantity in terms of which a necessary condition for separability (equivalently, a sufficient condition for entanglement) can be expressed. As we have seen, this quantity is the sum of the Schmidt coefficients of a bipartite state. It is quite natural to wonder whether the whole set of the Schmidt coefficients of a bipartite state may allow a stronger characterization of entanglement with respect to the RC alone. Otherwise stated, it is natural to look for further separability criteria relying only on the Schmidt equivalence class of a state. The issue is how to extract, if possible, the relevant information content from the Schmidt coefficients. Recently, in ref. [10], it has been proposed to consider the (elementary) symmetric polynomials in the Schmidt 
coefficients of a state $\rho \in \mathcal{D}(\mathcal{H})$, namely:

$$
\begin{array}{rlr}
\mathrm{M}^{[1]}(|\tilde{\rho}|) & =\sum_{a=1}^{\mathrm{d}} \mu_{a}, & \text { (first order symmetric polynomial) } \\
\mathrm{M}^{[2]}(|\tilde{\rho}|) & =\sum_{a<b} \mu_{a} \mu_{b}, \\
& \cdots \\
\mathrm{M}^{[l]}(|\tilde{\rho}|) & =\sum_{a_{1}<a_{2}<\cdots<a_{l}} \mu_{a_{1}} \mu_{a_{2}} \ldots \mu_{a_{l}}, \\
& \cdots \prod_{a=1}^{\mathrm{d}} \mu_{a} . \quad \text { (d-th order symmetric polynomial) }
\end{array}
$$

Note that we are regarding these polynomials as functions of the absolute value $|\tilde{\rho}|$ of the matrix $\tilde{\rho}$ associated with the state $\rho$, see (8) (the specific choice of the local bases is irrelevant). In fact, as we have seen, the Schmidt coefficients of $\rho$ coincide with the singular values of the matrix $\tilde{\rho}$; equivalently, with the eigenvalues of the positive square matrix $|\tilde{\rho}|=\sqrt{\tilde{\rho}^{\dagger} \tilde{\rho}}$. Therefore, the quantities $\mathrm{M}^{[1]}(|\tilde{\rho}|), \mathrm{M}^{[2]}(|\tilde{\rho}|), \ldots, \mathrm{M}^{[\mathrm{d}]}(|\tilde{\rho}|)$ appear as coefficients in the characteristic polynomial of the matrix $|\tilde{\rho}|$ :

$$
\chi_{|\tilde{\rho}|}(x)=\operatorname{det}(|\tilde{\rho}|-x \mathbb{I})=(-x)^{\mathrm{d}}+\sum_{l=1}^{\mathrm{d}} \mathrm{M}^{[l]}(|\tilde{\rho}|)(-x)^{\mathrm{d}-l} .
$$

In particular, the RC is based on the evaluation of an upper bound - on the set of separable states - for the symmetric polynomial of degree one $\mathrm{M}^{[1]}(|\tilde{\rho}|)$ (i.e. $\mathrm{M}^{[1]}(|\tilde{\rho}|) \leq 1$, for $\rho$ separable).

It is then natural to look for analogous upper bounds for the higher order symmetric polynomials. By a naive reasoning, we can say that, if the sum of the $\mathrm{d}$ Schmidt coefficients equals $S$, their product is upper bounded by $(S / \mathrm{d})^{\mathrm{d}}$. Hence we have the following necessary condition for the separability of a density operator $\rho \in \mathcal{D}(\mathcal{H})$ :

$$
\rho \text { separable } \Rightarrow \mathrm{M}^{[\mathrm{d}]}(|\tilde{\rho}|) \leq\left(\frac{1}{\mathrm{~d}}\right)^{\mathrm{d}} .
$$

It is clear that this separability criterion is weaker than the RC. In general, one can consider the symmetric polynomial of degree $l$ and obtain the following necessary conditions for the separability:

$$
\rho \text { separable } \Rightarrow \mathrm{M}^{[l]}(|\tilde{\rho}|) \leq\left(\begin{array}{l}
\mathrm{d} \\
l
\end{array}\right)\left(\frac{1}{\mathrm{~d}}\right)^{l} .
$$

A straightforward refinement of this criterion consists in taking into account the Schmidt rank R (recall the second assertion of Remark 2.1 $-1 \leq \mathrm{R} \leq \mathrm{d}$ - of the operator $\rho$; thus, we can write the following conditions:

$$
\rho \text { separable } \Rightarrow \mathrm{M}^{[l]}(|\tilde{\rho}|) \leq\left\{\begin{array}{cl}
\left(\begin{array}{c}
\mathrm{R} \\
l
\end{array}\right)\left(\frac{1}{\mathrm{R}}\right)^{l} & \text { if } l \leq \mathrm{R} \\
0 & \text { if } l>\mathrm{R}
\end{array},\right.
$$

$l=1, \ldots, \mathrm{d}$. Of course, for $l=1$, we have the RC. For $l \neq 1, l \leq \mathrm{R}$, the necessary conditions (27) are consequences of the RC; hence, they are weaker separability criteria. The existence of upper bounds, for the symmetric 
polynomials of degree $l \geq 2$, stricter than the 'naive' bounds in (27) — in particular, stricter upper bounds that may give rise to new separability criteria independent on the $\mathrm{RC}$ — has been investigated [10]. Numerical tests indicate that such stricter upper bounds indeed exist, but, till now, an analytical estimation has not been obtained. On our opinion, this is another interesting open problem.

\section{The symmetric polynomials in the Schmidt coefficients and the entanglement breaking quantum channels}

The RC - as well as the derived separability criteria based on the symmetric polynomials in the Schmidt coefficients discussed in the previous section - can be applied to the study of quantum channels, i.e. of completely positive trace-preserving (CPT) maps by exploiting the well known correspondence between quantum channels and quantum states (see [18, 19, 20]).

We will consider, as above, a pair of quantum systems with carrier Hilbert spaces $\mathcal{H}_{\mathrm{A}}$ and $\mathcal{H}_{\mathrm{B}}$, and we will denote by $\operatorname{CPT}\left(\mathcal{H}_{B}, \mathcal{H}_{A}\right)$ the set of CPT maps from the system $B$ to system $A$; i.e. from $\mathcal{L}\left(\mathcal{H}_{B}\right)$ into $\mathcal{L}\left(\mathcal{H}_{\mathrm{A}}\right)$. Given a CPT map $\mathcal{E} \in \operatorname{CPT}\left(\mathcal{H}_{\mathrm{B}}, \mathcal{H}_{\mathrm{A}}\right)$, one can associate with $\mathcal{E}$ a state $\rho_{\mathcal{E}} \in \mathcal{D}\left(\mathcal{H}_{\mathrm{A}} \otimes \mathcal{H}_{\mathrm{B}}\right)$ in the following canonical way:

$$
\mathcal{E} \longmapsto \rho_{\mathcal{E}}=(\mathcal{E} \otimes \mathcal{I})\left(\rho_{\mathcal{I}}\right),
$$

with $\rho_{\mathcal{I}}=|\psi\rangle\langle\psi| \in \mathcal{D}\left(\mathcal{H}_{\mathrm{B}} \otimes \mathcal{H}_{\mathrm{B}}\right)$ denoting a maximally entangled (pure) state - namely, $|\psi\rangle=\frac{1}{\sqrt{N_{\mathrm{B}}}} \sum_{\alpha=1}^{N_{\mathrm{B}}}|\alpha\rangle \otimes$ $|\alpha\rangle$, for some orthonormal basis $\{|\alpha\rangle\}_{\alpha=1}^{N_{\mathrm{B}}}$ in $\mathcal{H}_{\mathrm{B}}$ - and $\mathcal{I}$ denoting the identity in $\mathcal{L}\left(\mathcal{H}_{\mathrm{B}}\right)$.

A quantum channel $\mathcal{E} \in \mathrm{CPT}\left(\mathcal{H}_{\mathrm{B}}, \mathcal{H}_{\mathrm{A}}\right)$ is said to be entanglement breaking (EB) if the extended map $\mathcal{E} \otimes \mathcal{I}$ transforms any state into a separable one [21]. One can show — see [21] — that a CPT is EB if and only if $\mathcal{E} \otimes \mathcal{I}$ maps a maximally entangled state into a separable one; hence, if and only if the canonically associated state is separable. In particular, a separability criterion also gives a necessary conditions for a channel to be entanglement breaking. The RC and the derived criteria based on symmetric polynomials in the Schmidt coefficients have an interesting interpretation when considered as criteria for EB channels.

In fact, choose local orthonormal bases $\left\{F_{a}^{\mathrm{A}} \mid a=1, \ldots, N_{\mathrm{A}}^{2}\right\},\left\{F_{\alpha}^{\mathrm{B}} \mid \alpha=1, \ldots, N_{\mathrm{B}}^{2}\right\}$ in $\mathcal{L}\left(\mathcal{H}_{\mathrm{A}}\right)$ and $\mathcal{L}\left(\mathcal{H}_{\mathrm{B}}\right)$, respectively, and consider the 'matrix elements'

$$
\tilde{\mathcal{E}}_{a \alpha}=\operatorname{tr}\left(F_{a}^{\mathrm{A}^{\dagger}} \mathcal{E}\left(F_{\alpha}^{\mathrm{B}^{\dagger}}\right)\right)
$$

It is easy to check that the canonically associated density matrix $\rho_{\mathcal{E}}$ has the following form:

$$
\rho_{\mathcal{E}}=\frac{1}{N_{\mathrm{B}}} \tilde{\mathcal{E}}_{a \alpha} F_{a}^{\mathrm{A}} \otimes F_{\alpha}^{\mathrm{B}}
$$

Then, we can consider the characteristic polynomial

$$
\chi_{\left|\tilde{\rho}_{\mathcal{E}}\right|}(x)=\operatorname{det}\left(\frac{1}{N_{\mathrm{B}}}|\tilde{\mathcal{E}}|-x \mathbb{I}\right)=(-x)^{\mathrm{d}}+\sum_{l=1}^{\mathrm{d}} \mathrm{M}^{[l]}(|\tilde{\mathcal{E}}|)\left(\frac{1}{N_{\mathrm{B}}}\right)^{l}(-x)^{\mathrm{d}-l} .
$$

Relation (31) establishes a link between the symmetric polynomials in the singular values of the matrix $\tilde{\mathcal{E}}$ (the eigenvalues of $|\tilde{\mathcal{E}}|$ ) and the symmetric polynomials in the Schmidt coefficients of the state $\rho_{\mathcal{E}}$. Thus, one can apply the results of the preceding section. 
Let us consider, for instance, the case where the matrix $\tilde{\mathcal{E}}$ has full rank. Then, one obtains from relation (25) the following necessary condition for the channel $\mathcal{E}$ to be entanglement breaking:

$$
\mathcal{E} \text { entanglement breaking } \Leftrightarrow \rho_{\mathcal{E}} \text { separable } \Rightarrow \operatorname{det}(|\tilde{\mathcal{E}}|) \leq\left(\frac{N_{\mathrm{B}}}{\mathrm{d}}\right)^{\mathrm{d}} \text {. }
$$

This result relates a geometric property of the map — such as the rate of contraction of volume, which equals $\operatorname{det}(|\tilde{\mathcal{E}}|)$ - to the property of being entanglement breaking. In general, relation (27) implies that, if the matrix $\tilde{\mathcal{E}}$ has rank R, we can write the following necessary conditions for the channel $\mathcal{E}$ to be entanglement breaking:

$$
\mathcal{E} \text { entanglement breaking } \Rightarrow \mathrm{M}^{[l]}(|\tilde{\mathcal{E}}|) \leq\left(\begin{array}{c}
\mathrm{R} \\
l
\end{array}\right)\left(\frac{N_{\mathrm{B}}}{\mathrm{R}}\right)^{l},
$$

for $l=1, \ldots, \mathrm{R}$.

\section{Entanglement and the Schmidt coefficients of transformed operators}

As we have seen in the preceding sections, one can fruitfully extract some 'information' from the Schmidt coefficients for detecting entanglement. In particular, the RC relies on the sum of the Schmidt coefficients of a bipartite state (which coincides with the CCN of the state). A slightly more sophisticated strategy is the following. Instead of considering the Schmidt coefficients of the state under scrutiny itself, one may consider the Schmidt coefficients of a 'transformed operator' suitably associated with this state. Such a transformed operator will not be, in general, a density operator. However, one may heuristically regard this transformation as an 'entanglement highlighting procedure' preliminary to the entanglement detection via the evaluation of the $\mathrm{CCN}$.

Let $\rho \in \mathcal{D}(\mathcal{H})$ be a separable state in the bipartite Hilbert space $\mathcal{H}=\mathcal{H}_{\mathrm{A}} \otimes \mathcal{H}_{\mathrm{B}}$, with a separability decomposition of the form

$$
\rho=\sum_{i} p_{i} \rho_{i}^{\mathrm{A}} \otimes \rho_{i}^{\mathrm{B}}
$$

where $\sum_{i} p_{i}=1$ and $p_{i}>0$. Let us denote by $\rho_{\mathrm{A}}, \rho_{\mathrm{B}}$ the marginals (reduced density operators) of $\rho$, namely:

$$
\rho_{\mathrm{A}}:=\operatorname{tr}_{\mathrm{B}}(\rho)=\sum_{i} p_{i} \rho_{i}^{\mathrm{A}}, \quad \rho_{\mathrm{B}}:=\operatorname{tr}_{\mathrm{A}}(\rho)=\sum_{i} p_{i} \rho_{i}^{\mathrm{B}}
$$

In order to associate with $\rho$ a suitable 'transformed operator', for each of the two subsystems A and B we will consider a pair of linear or antilinear (super)operators. Precisely, we will consider a set of operators

$$
\left\{\mathfrak{E}_{1}^{\mathrm{A}}, \mathfrak{E}_{2}^{\mathrm{A}}: \mathcal{L}\left(\mathcal{H}_{\mathrm{A}}\right) \rightarrow \mathcal{L}\left(\mathcal{H}_{\mathrm{A}}\right), \mathfrak{E}_{1}^{\mathrm{B}}, \mathfrak{E}_{2}^{\mathrm{B}}: \mathcal{L}\left(\mathcal{H}_{\mathrm{B}}\right) \rightarrow \mathcal{L}\left(\mathcal{H}_{\mathrm{B}}\right)\right\}
$$

that are assumed to be jointly linear or antilinear - i.e. either all linear or all antilinear - in such a way that one can consistently define tensor products and sums of these operators. We will associate with $\rho$ the operator $\mathfrak{E}_{1,2}^{\mathrm{A}, \mathrm{B}}(\rho) \in \mathcal{L}(\mathcal{H})$ defined as

$$
\mathfrak{E}_{1,2}^{\mathrm{A}, \mathrm{B}}(\rho):=\frac{1}{2}\left(\mathfrak{E}_{1}^{\mathrm{A}} \otimes \mathfrak{E}_{1}^{\mathrm{B}}+\mathfrak{E}_{2}^{\mathrm{A}} \otimes \mathfrak{E}_{2}^{\mathrm{B}}\right)(\rho)+\frac{1}{2}\left(\mathfrak{E}_{1}^{\mathrm{A}} \otimes \mathfrak{E}_{2}^{\mathrm{B}}+\mathfrak{E}_{2}^{\mathrm{A}} \otimes \mathfrak{E}_{1}^{\mathrm{B}}\right)\left(\rho_{\mathrm{A}} \otimes \rho_{\mathrm{B}}\right) .
$$


We stress that, in general, $\mathfrak{E}_{1,2}^{\mathrm{A}, \mathrm{B}}(\rho)$ is not a density operator. It is easy to check that

$$
\begin{aligned}
\mathfrak{E}_{1,2}^{\mathrm{A}, \mathrm{B}}(\rho) & =\frac{1}{2} \sum_{i, j} p_{i} p_{j}\left(\mathfrak{E}_{1}^{\mathrm{A}}\left(\rho_{i}^{\mathrm{A}}\right)+\mathfrak{E}_{2}^{\mathrm{A}}\left(\rho_{j}^{\mathrm{A}}\right)\right) \otimes\left(\mathfrak{E}_{1}^{\mathrm{B}}\left(\rho_{i}^{\mathrm{B}}\right)+\mathfrak{E}_{2}^{\mathrm{B}}\left(\rho_{j}^{\mathrm{B}}\right)\right) \\
& \equiv \frac{1}{2} \sum_{i, j} p_{i} p_{j}\left(\mathfrak{E}_{1}^{\mathrm{A}}\left(\rho_{i}^{\mathrm{A}}\right)+\mathfrak{E}_{2}^{\mathrm{A}}\left(\rho_{j}^{\mathrm{A}}\right)\right) \otimes(\mathrm{A} \rightarrow \mathrm{B}),
\end{aligned}
$$

where the symbol $(A \rightarrow B)$ denotes repetition of the preceding term with the substitution of the subsystem $A$ with the subsystem $B$ (all the rest remaining unchanged). At this point, one can try to obtain an estimate of the CCN of the transformed operator $\mathfrak{E}_{1,2}^{\mathrm{A}, \mathrm{B}}(\rho)$. Using relation (38) and the triangle inequality, one finds out that

$$
\begin{aligned}
\left\|\mathfrak{E}_{1,2}^{\mathrm{A}, \mathrm{B}}(\rho)\right\|_{\mathrm{CCN}} & =\frac{1}{2}\left\|\sum_{i, j} p_{i} p_{j}\left(\mathfrak{E}_{1}^{\mathrm{A}}\left(\rho_{i}^{\mathrm{A}}\right)+\mathfrak{E}_{2}^{\mathrm{A}}\left(\rho_{j}^{\mathrm{A}}\right)\right) \otimes(\mathrm{A} \rightarrow \mathrm{B})\right\|_{\mathrm{CCN}} \\
& \leq \frac{1}{2} \sum_{i, j} p_{i} p_{j}\left\|\left(\mathfrak{E}_{1}^{\mathrm{A}}\left(\rho_{i}^{\mathrm{A}}\right)+\mathfrak{E}_{2}^{\mathrm{A}}\left(\rho_{j}^{\mathrm{A}}\right)\right) \otimes(\mathrm{A} \rightarrow \mathrm{B})\right\|_{\mathrm{CCN}} .
\end{aligned}
$$

Next, recall that the CCN $\|\cdot\|_{\mathrm{CCN}}$ is a cross norm on $\mathcal{L}(\mathcal{H})$; hence:

$$
\begin{aligned}
\left\|\left(\mathfrak{E}_{1}^{\mathrm{A}}\left(\rho_{i}^{\mathrm{A}}\right)+\mathfrak{E}_{2}^{\mathrm{A}}\left(\rho_{j}^{\mathrm{A}}\right)\right) \otimes(\mathrm{A} \rightarrow \mathrm{B})\right\|_{\mathrm{CCN}} & =\left\|\mathfrak{E}_{1}^{\mathrm{A}}\left(\rho_{i}^{\mathrm{A}}\right)+\mathfrak{E}_{2}^{\mathrm{A}}\left(\rho_{j}^{\mathrm{A}}\right)\right\|_{\mathrm{HS}}\left\|\mathfrak{E}_{1}^{\mathrm{B}}\left(\rho_{i}^{\mathrm{B}}\right)+\mathfrak{E}_{2}^{\mathrm{B}}\left(\rho_{j}^{\mathrm{B}}\right)\right\|_{\mathrm{HS}} \\
& =\left(\left\langle\mathfrak{E}_{1}^{\mathrm{A}}\left(\rho_{i}^{\mathrm{A}}\right), \mathfrak{E}_{1}^{\mathrm{A}}\left(\rho_{i}^{\mathrm{A}}\right)\right\rangle_{\mathrm{HS}}+\left\langle\mathfrak{E}_{2}^{\mathrm{A}}\left(\rho_{j}^{\mathrm{A}}\right), \mathfrak{E}_{2}^{\mathrm{A}}\left(\rho_{j}^{\mathrm{A}}\right)\right\rangle_{\mathrm{HS}}\right. \\
& \left.+\left(\left\langle\mathfrak{E}_{1}^{\mathrm{A}}\left(\rho_{i}^{\mathrm{A}}\right), \mathfrak{E}_{2}^{\mathrm{A}}\left(\rho_{j}^{\mathrm{A}}\right)\right\rangle_{\mathrm{HS}}+\text { c.c. }\right)\right)^{\frac{1}{2}}(\mathrm{~A} \rightarrow \mathrm{B})^{\frac{1}{2}} .
\end{aligned}
$$

Therefore, if we assume that - for some $\epsilon^{\mathrm{A}}, \epsilon^{\mathrm{B}} \geq 0$ - the operators $\mathfrak{E}_{1}^{\mathrm{A}}, \mathfrak{E}_{2}^{\mathrm{A}}, \mathfrak{E}_{1}^{\mathrm{B}}, \mathfrak{E}_{2}^{\mathrm{B}}$ satisfy the conditions

$$
\left\|\mathfrak{E}_{1}^{\mathrm{A}}\left(\hat{\sigma}_{1}^{\mathrm{A}}\right)\right\|_{\mathrm{HS}}^{2}+\left\|\mathfrak{E}_{2}^{\mathrm{A}}\left(\hat{\sigma}_{2}^{\mathrm{A}}\right)\right\|_{\mathrm{HS}}^{2} \leq 2 \epsilon^{\mathrm{A}}, \quad\left\|\mathfrak{E}_{1}^{\mathrm{B}}\left(\hat{\sigma}_{1}^{\mathrm{B}}\right)\right\|_{\mathrm{HS}}^{2}+\left\|\mathfrak{E}_{2}^{\mathrm{B}}\left(\hat{\sigma}_{2}^{\mathrm{B}}\right)\right\|_{\mathrm{HS}}^{2} \leq 2 \epsilon^{\mathrm{B}}
$$

$\forall \hat{\sigma}_{1}^{\mathrm{A}}, \hat{\sigma}_{2}^{\mathrm{A}} \in \mathcal{D}\left(\mathcal{H}_{\mathrm{A}}\right), \forall \hat{\sigma}_{1}^{\mathrm{B}}, \hat{\sigma}_{2}^{\mathrm{B}} \in \mathcal{D}\left(\mathcal{H}_{\mathrm{B}}\right)$, we find the following estimate:

$$
\left\|\left(\mathfrak{E}_{1}^{\mathrm{A}}\left(\rho_{i}^{\mathrm{A}}\right)+\mathfrak{E}_{2}^{\mathrm{A}}\left(\rho_{j}^{\mathrm{A}}\right)\right) \otimes(\mathrm{A} \rightarrow \mathrm{B})\right\|_{\mathrm{CCN}} \leq 2 \sqrt{\left(\epsilon^{\mathrm{A}}+\frac{1}{2}\left(\left\langle\mathfrak{E}_{1}^{\mathrm{A}}\left(\rho_{i}^{\mathrm{A}}\right), \mathfrak{E}_{2}^{\mathrm{A}}\left(\rho_{j}^{\mathrm{A}}\right)\right\rangle_{\mathrm{HS}}+\text { c.c. }\right)\right)} \sqrt{(\mathrm{A} \rightarrow \mathrm{B})} .
$$

Observe that the same estimate holds true if one replaces the cross norm $\|\cdot\|_{\mathrm{CCN}}$ with any subcross norm in $\mathcal{L}(\mathcal{H})$, i.e. with a norm $\|\cdot\|_{\mathrm{suB}}: \mathcal{L}(\mathcal{H}) \rightarrow \mathbb{R}^{+}$such that

$$
\|A \otimes B\|_{\mathrm{SUB}} \leq\|A\|_{\mathrm{HS}}\|B\|_{\mathrm{HS}}, \quad \forall A \in \mathcal{L}\left(\mathcal{H}_{\mathrm{A}}\right), \forall B \in \mathcal{L}\left(\mathcal{H}_{\mathrm{B}}\right)
$$

Eventually, from inequalities (39) and (42), we obtain:

$$
\begin{aligned}
\left\|\mathfrak{E}_{1,2}^{\mathrm{A}, \mathrm{B}}(\rho)\right\|_{\mathrm{CCN}} & \leq \sum_{i, j} \sqrt{p_{i} p_{j}\left(\epsilon^{\mathrm{A}}+\frac{1}{2}\left(\left\langle\mathfrak{E}_{1}^{\mathrm{A}}\left(\rho_{i}^{\mathrm{A}}\right), \mathfrak{E}_{2}^{\mathrm{A}}\left(\rho_{j}^{\mathrm{A}}\right)\right\rangle_{\mathrm{HS}}+\text { c.c. }\right)\right)} \\
& \times \sqrt{p_{i} p_{j}\left(\epsilon^{\mathrm{B}}+\frac{1}{2}\left(\left\langle\mathfrak{E}_{1}^{\mathrm{B}}\left(\rho_{i}^{\mathrm{B}}\right), \mathfrak{E}_{2}^{\mathrm{B}}\left(\rho_{j}^{\mathrm{B}}\right)\right\rangle_{\mathrm{HS}}+\text { c.c. }\right)\right)} \\
& \leq \sqrt{\left(\epsilon^{\mathrm{A}}+\frac{1}{2}\left(\sum_{i, j} p_{i} p_{j}\left\langle\mathfrak{E}_{1}^{\mathrm{A}}\left(\rho_{i}^{\mathrm{A}}\right), \mathfrak{E}_{2}^{\mathrm{A}}\left(\rho_{j}^{\mathrm{A}}\right)\right\rangle_{\mathrm{HS}}+\text { c.c. }\right)\right)} \sqrt{(\mathrm{A} \rightarrow \mathrm{B})}
\end{aligned}
$$


where the second inequality above follows from the Cauchy-Schwarz inequality. In conclusion, for every separable state $\rho \in \mathcal{D}(\mathcal{H})$, the following inequality holds:

$$
\left\|\mathfrak{E}_{1,2}^{\mathrm{A}, \mathrm{B}}(\rho)\right\|_{\mathrm{CCN}} \leq \sqrt{\left(\epsilon^{\mathrm{A}}+\frac{1}{2}\left(\left\langle\mathfrak{E}_{1}^{\mathrm{A}}\left(\rho_{\mathrm{A}}\right), \mathfrak{E}_{2}^{\mathrm{A}}\left(\rho_{\mathrm{A}}\right)\right\rangle_{\mathrm{HS}}+\text { c.c. }\right)\right)} \sqrt{(\mathrm{A} \rightarrow \mathrm{B})} .
$$

The previous result can be generalized as follows. For any $n \geq 1$, consider a set of $2 n$ jointly linear or antilinear operators

$$
\left\{\mathfrak{E}_{1}^{\mathrm{A}}, \ldots, \mathfrak{E}_{\mathrm{n}}^{\mathrm{A}}: \mathcal{L}\left(\mathcal{H}_{\mathrm{A}}\right) \rightarrow \mathcal{L}\left(\mathcal{H}_{\mathrm{A}}\right), \mathfrak{E}_{1}^{\mathrm{B}}, \ldots, \mathfrak{E}_{\mathrm{n}}^{\mathrm{B}}: \mathcal{L}\left(\mathcal{H}_{\mathrm{B}}\right) \rightarrow \mathcal{L}\left(\mathcal{H}_{\mathrm{B}}\right)\right\}
$$

These operators allow to define a map

$$
\mathfrak{E}_{1, \ldots, \mathrm{n}}^{\mathrm{A}, \mathrm{B}}: \mathcal{D}(\mathcal{H}) \rightarrow \mathcal{L}(\mathcal{H})
$$

by setting:

$$
\mathfrak{E}_{1, \ldots, \mathrm{n}}^{\mathrm{A}, \mathrm{B}}(\rho):=\mathrm{n}^{-1}\left(\mathfrak{E}_{1}^{\mathrm{A}} \otimes \mathfrak{E}_{1}^{\mathrm{B}}+\cdots+\mathfrak{E}_{\mathrm{n}}^{\mathrm{A}} \otimes \mathfrak{E}_{\mathrm{n}}^{\mathrm{B}}\right)(\rho)+\mathrm{n}^{-1}\left(\sum_{k \neq l} \mathfrak{E}_{k}^{\mathrm{A}} \otimes \mathfrak{E}_{l}^{\mathrm{B}}\right)\left(\rho_{\mathrm{A}} \otimes \rho_{\mathrm{B}}\right), \quad \forall \rho \in \mathcal{D}(\mathcal{H}),
$$

where $\rho_{\mathrm{A}}$ and $\rho_{\mathrm{B}}$ are the marginals of $\rho$, namely: $\rho_{\mathrm{A}}:=\operatorname{tr}_{\mathrm{B}}(\rho), \rho_{\mathrm{B}}:=\operatorname{tr}_{\mathrm{A}}(\rho)$. We will call the operator $\mathfrak{E}_{1, \ldots, \mathrm{n}}^{\mathrm{A}, \mathrm{B}}(\rho)$ the $\mathfrak{E}_{1, \ldots, \mathrm{n}}^{\mathrm{A}, \mathrm{B}}$-transform of the bipartite state $\rho$. Then, the following result holds (see [9]):

Theorem 6.1 Let $\mathfrak{E}_{1}^{\mathrm{A}}, \ldots, \mathfrak{E}_{\mathrm{n}}^{\mathrm{A}}: \mathcal{L}\left(\mathcal{H}_{\mathrm{A}}\right) \rightarrow \mathcal{L}\left(\mathcal{H}_{\mathrm{A}}\right), \mathfrak{E}_{1}^{\mathrm{B}}, \ldots, \mathfrak{E}_{\mathrm{n}}^{\mathrm{B}}: \mathcal{L}\left(\mathcal{H}_{\mathrm{B}}\right) \rightarrow \mathcal{L}\left(\mathcal{H}_{\mathrm{B}}\right)$ be jointly linear or antilinear operators that, for some $\epsilon^{\mathrm{A}}, \epsilon^{\mathrm{B}} \geq 0$, satisfy the following conditions:

$$
\begin{gathered}
\left\|\mathfrak{E}_{1}^{\mathrm{A}}\left(\hat{\sigma}_{1}^{\mathrm{A}}\right)\right\|_{\mathrm{HS}}^{2}+\cdots+\left\|\mathfrak{E}_{\mathrm{n}}^{\mathrm{A}}\left(\hat{\sigma}_{\mathrm{n}}^{\mathrm{A}}\right)\right\|_{\mathrm{HS}}^{2} \leq \mathrm{n} \epsilon^{\mathrm{A}}, \quad \forall \hat{\sigma}_{1}^{\mathrm{A}}, \ldots, \hat{\sigma}_{\mathrm{n}}^{\mathrm{A}} \in \mathcal{D}\left(\mathcal{H}_{\mathrm{A}}\right), \\
\left\|\mathfrak{E}_{1}^{\mathrm{B}}\left(\hat{\sigma}_{1}^{\mathrm{B}}\right)\right\|_{\mathrm{HS}}^{2}+\cdots+\left\|\mathfrak{E}_{\mathrm{n}}^{\mathrm{B}}\left(\hat{\sigma}_{\mathrm{n}}^{\mathrm{B}}\right)\right\|_{\mathrm{HS}}^{2} \leq \mathrm{n} \epsilon^{\mathrm{B}}, \quad \forall \hat{\sigma}_{1}^{\mathrm{B}}, \ldots, \hat{\sigma}_{\mathrm{n}}^{\mathrm{B}} \in \mathcal{D}\left(\mathcal{H}_{\mathrm{B}}\right) .
\end{gathered}
$$

Then, for every separable state $\rho \in \mathcal{D}(\mathcal{H})$, the $\mathfrak{E}_{1, \ldots, \mathrm{n}}^{\mathrm{A}, \mathrm{B}}$-transform of $\rho$ satisfies the following inequality:

$$
\left\|\mathfrak{E}_{1, \ldots, \mathrm{n}}^{\mathrm{A}, \mathrm{B}}(\rho)\right\|_{\mathrm{CCN}} \leq \sqrt{\left(\epsilon^{\mathrm{A}}+\frac{1}{\mathrm{n}} \sum_{k<l}\left(\left\langle\mathfrak{E}_{k}^{\mathrm{A}}\left(\rho_{\mathrm{A}}\right), \mathfrak{E}_{l}^{\mathrm{A}}\left(\rho_{\mathrm{A}}\right)\right\rangle_{\mathrm{HS}}+\text { c.c. }\right)\right)(\mathrm{A} \rightarrow \mathrm{B})} .
$$

Remark 6.2 As already noticed, on the l.h.s. of inequality (50) we may replace the norm $\|\cdot\|_{\mathrm{CCN}}$ with any subcross norm in $\mathcal{L}(\mathcal{H})$. Such a replacement may give rise to further separability criteria that would be, however, weaker than the corresponding separability criteria involving the CCN. Indeed, it is easy to show that

$$
\|C\|_{\mathrm{SUB}} \leq\|C\|_{\mathrm{CCN}}, \quad \forall C \in \mathcal{L}(\mathcal{H})
$$

for every subcross norm $\|\cdot\|_{\mathrm{SUB}}$ in $\mathcal{L}(\mathcal{H}) .3$

Observe that Theorem 6.1 induces a class of separability criteria that can be regarded as a generalization of the RC. Indeed, for $\mathrm{n}=1$, and $\mathfrak{E}_{1}^{\mathrm{A}}, \mathfrak{E}_{1}^{\mathrm{B}}$ coinciding with the identity superoperators (so that we can set: $\epsilon^{\mathrm{A}}=\epsilon^{\mathrm{B}}=1$ ),

${ }^{3}$ Let $\sum_{k=1}^{n} A_{k} \otimes B_{k}$ any decomposition of $C \in \mathcal{L}(\mathcal{H})$. Then, by the triangle inequality and the subcross property of $\|\cdot\|$ SuB we get : $\|C\|_{\mathrm{SUB}} \leq \sum_{k=1}^{n}\left\|A_{k} \otimes B_{k}\right\|_{\mathrm{SUB}} \leq \sum_{k=1}^{n}\left\|A_{k}\right\|_{\mathrm{HS}}\left\|B_{k}\right\|_{\mathrm{HS}}$. From the definition of the CCN inequality [51] follows. 
we recover the RC (22). Notice also that conditions (48)-(49) are satisfied - with $\epsilon^{\mathrm{A}}=\epsilon^{\mathrm{B}}=1-$ if the superoperators $\left\{\mathfrak{E}_{k}^{\mathrm{A}}, \mathfrak{E}_{k}^{\mathrm{B}}\right\}_{k=1, \ldots, \mathrm{n}}$ are such that

$$
\left\|\mathfrak{E}_{k}^{\mathrm{A}}\left(\hat{\sigma}^{\mathrm{A}}\right)\right\|_{\mathrm{HS}} \leq 1,\left\|\mathfrak{E}_{k}^{\mathrm{B}}\left(\hat{\sigma}^{\mathrm{B}}\right)\right\|_{\mathrm{HS}} \leq 1, \quad k=1, \ldots, \mathrm{n},
$$

$\forall \hat{\sigma}^{\mathrm{A}} \in \mathcal{D}\left(\mathcal{H}_{\mathrm{A}}\right), \forall \hat{\sigma}^{\mathrm{B}} \in \mathcal{D}\left(\mathcal{H}_{\mathrm{B}}\right)$. One can assume, in particular, that they are trace-norm-nonincreasing on positive operators (since the Hilbert-Schmidt norm is majorized by the trace norm); for instance, positive trace-preserving linear maps.

For $\mathrm{n}=2-$ with the following choice of the superoperators:

$$
\mathfrak{E}_{1}^{\mathrm{A}}=e^{\mathrm{i} \theta} \mathcal{I}^{\mathrm{A}}, \quad \mathfrak{E}_{1}^{\mathrm{B}}=e^{-\mathrm{i} \theta} \mathcal{I}^{\mathrm{B}}, \quad \mathfrak{E}_{2}^{\mathrm{A}}=-\mathcal{I}^{\mathrm{A}}, \quad \mathfrak{E}_{2}^{\mathrm{B}}=-\mathcal{I}^{\mathrm{B}}, \quad \theta \in[0, \pi],
$$

where $\mathcal{I}^{\mathrm{A}}, \mathcal{I}^{\mathrm{B}}$ denote the identity in $\mathcal{L}\left(\mathcal{H}_{\mathrm{A}}\right)$ and $\mathcal{L}\left(\mathcal{H}_{\mathrm{B}}\right)$, respectively — we obtain an interesting class of separability criteria. Namely, for every separable state $\rho \in \mathcal{D}(\mathcal{H})$, the following $\theta$-parametrized family of inequalities holds:

$$
\left\|\rho-\cos \theta \rho_{\mathrm{A}} \otimes \rho_{\mathrm{B}}\right\|_{\mathrm{CCN}} \leq \sqrt{\left(1-\cos \theta \operatorname{tr}\left(\rho_{\mathrm{A}}^{2}\right)\right)\left(1-\cos \theta \operatorname{tr}\left(\rho_{\mathrm{B}}^{2}\right)\right)}, \quad \theta \in[0, \pi] .
$$

In particular, for $\theta=\pi / 2$, we re-obtain once again the RC. For $\theta=\pi$, we recover a separability criterion recently discovered by Zhang et al., see ref. [22]. This criterion has been shown to be stronger than the RC.

Another interesting class of separability criteria contained in the family of criteria induced by Theorem 6.1 are the so-called 'local filtering enhancements' of the RC, see [9, 23]. In this case, we have $\mathrm{n}=2$ and

$$
\begin{gathered}
\mathfrak{E}_{1}^{\mathrm{A}}: \mathcal{L}\left(\mathcal{H}_{\mathrm{A}}\right) \ni A \mapsto L^{\mathrm{A}} A\left(L^{\mathrm{A}}\right)^{\dagger} \in \mathcal{L}\left(\mathcal{H}_{\mathrm{A}}\right), \mathfrak{E}_{1}^{\mathrm{B}}: \mathcal{L}\left(\mathcal{H}_{\mathrm{B}}\right) \ni B \mapsto L^{\mathrm{B}} B\left(L^{\mathrm{B}}\right)^{\dagger} \in \mathcal{L}\left(\mathcal{H}_{\mathrm{B}}\right), \\
\mathfrak{E}_{2}^{\mathrm{A}}: \mathcal{L}\left(\mathcal{H}_{\mathrm{A}}\right) \ni A \mapsto \mathrm{i} \mathfrak{E}_{1}^{\mathrm{A}}(A) \in \mathcal{L}\left(\mathcal{H}_{\mathrm{A}}\right), \mathfrak{E}_{2}^{\mathrm{B}}: \mathcal{L}\left(\mathcal{H}_{\mathrm{B}}\right) \ni B \mapsto-\mathrm{i} \mathfrak{E}_{1}^{\mathrm{B}}(B) \in \mathcal{L}\left(\mathcal{H}_{\mathrm{B}}\right),
\end{gathered}
$$

where $L^{\mathrm{A}}, L^{\mathrm{B}}$ are suitable linear operators in $\mathcal{L}\left(\mathcal{H}_{\mathrm{A}}\right)$ and $\mathcal{L}\left(\mathcal{H}_{\mathrm{B}}\right)$, respectively (such that we can set: $\epsilon^{\mathrm{A}}=\epsilon^{\mathrm{B}}=1$ ); hence, for every separable state $\rho \in \mathcal{D}(\mathcal{H})$,

$$
\left\|\left(L^{\mathrm{A}} \otimes L^{\mathrm{B}}\right) \rho\left(L^{\mathrm{A}} \otimes L^{\mathrm{B}}\right)^{\dagger}\right\|_{\mathrm{CCN}} \leq 1
$$

'Optimal choices' of the the operators $L^{\mathrm{A}}, L^{\mathrm{B}}$ (for a given state $\rho$ under scrutiny) can be achieved by means of a constructive procedure, see [23] and references therein. It would be interesting to investigate the possibility of extending such a procedure to a broader class of separability criteria induced by inequality (50).

\section{Conclusions}

The detection of the entanglement of a bipartite state is a formidable problem that is nowadays the object of intense investigation by a broad scientific community. Among the several separability criteria proposed so far in the literature, we think that a prominent position — at least from the conceptual point of view — is occupied by the realignment criterion ( $\mathrm{RC})$, which, as we have seen, admits two different formulations. In its more 'simple-minded' formulation [12] (that is, however, of very practical use) it does not reveal immediately its profound meaning, which is related to the computable cross norm (CCN) [13, 14]. The formulation of the RC in terms of the CCN - see Sect. 3 - brings under our attention the role played by the Schmidt coefficients of a bipartite state. In particular, the RC relies on the sum of the Schmidt coefficients (which coincides with the CCN of the state). However - see 
Sect. 4 - there is evidence that the whole set of the Schmidt coefficients (rather than their sum alone) may allow a stricter characterization of entanglement with respect to the RC. Furthermore, as shown in Sect. 6, one can adopt a more sophisticated strategy in order to extract information about entanglement from the Schmidt coefficients. This strategy consists in first suitably transforming a state and then considering the Schmidt coefficients of the transformed operator.

During our brief survey on the role of the Schmidt coefficients in the detection of entanglement, we have indicated various open problems. First, as mentioned in Sect. 2] it would be interesting to achieve a deeper understanding of the structure of the Schmidt equivalence classes of states; in particular, to ascertain whether they are differentiable manifolds seems to be a harsh mathematical problem that would deserve further attention. Next, as observed in Sect. 4, it would be desirable to extract from the Schmidt coefficients more information about entanglement than that revealed by their sum - i.e. by the $\mathrm{CCN}$ of a state - for instance, by means of suitable functions of these coefficients (e.g., the symmetric polynomials). In other words, it would be interesting to find further separability criteria relying on the Schmidt equivalence classes only. Finally, another interesting open question is the possibility of obtaining a further refinement of the entanglement detection strategy outlined in Sect. 6, with the aim of selecting 'optimal transformations' (with regard to the entanglement detection) for each given state under scrutiny, as in the special case of the 'local filtering enhancements' of the RC.

\section{Acknowledgments}

The main results of the paper were presented by the authors at the international conference The Jubilee 40th Symposium on Mathematical Physics - Geometry \& Quanta (25-28 June 2008, Torun, Poland). They wish to thank the organizers for their very kind hospitality.

\section{References}

[1] I. Bengtsson and K. Zyczkowski, Geometry of Quantum States, Cambridge University Press, Cambridge (2006).

[2] M.A. Nielsen and I.L. Chuang, Quantum Computation and Quantum Information, Cambridge University Press, Cambridge (2000).

[3] R. F. Werner, Phys. Rev. A 40 (1989), 4277.

[4] R. Horodecki, P. Horodecki, M. Horodecki, K. Horodecki, Quantum Entanglement, arXiv:quant-ph/0702225 (2007).

[5] U. Fano, Rev. Mod. Phys. 55 (1983), 855.

[6] G. Kimura, Phys. Lett. A 314 (2003), 339;

M. S. Byrd and N. Khaneja, Phys. Rev. A 68 (2003), 062322.

[7] J. I. de Vicente, Quantum Inf. Comput. 7 (2007), 624;

J. I. de Vicente, J. Phys. A: Math. Theor. 41 (2008), 065309. 
[8] S. Yu and N. Liu, Phys. Rev. Lett. 95 (2005), 150504;

O. Gühne, M. Mechler, G. Toth, P. Adam, Phys. Rev. A 74 (2006), 010301(R).

[9] P. Aniello, C. Lupo, J. Phys. A: Math. Theor. 41 (2008), 355303.

[10] C. Lupo, P. Aniello, A. Scardicchio J. Phys. A: Math. Theor. 41 (2008), 415301.

[11] R.A. Horn and C.R. Johnson, Topics in Matrix Analysis, Cambridge University Press, Cambridge (1991).

[12] K. Chen, L.-A. Wu, Quant. Inf. Comp. 3 (2003), 193.

[13] O. Rudolph, quant-ph/0202121 (2002).

[14] O. Rudolph, Phys. Rev. A 67 (2003), 032312.

[15] G. Vidal, J. Mod. Opt. 47 (2000), 355.

[16] A. Peres, Quantum Theory: Concepts and Methods, Kluwer Academic Publishers, Dordrecht (1993).

[17] M. M. Sinołęcka, K. Życzkowski, M. Kuś, Act. Phys. Pol. B 33 (2002), 2081.

[18] E. C. G. Sudarshan, P. M. Mathews and J. Rau, Phys. Rev. Lett. 121 (1961), 920.

[19] A. Jamiołkowski, Rep. Math. Phys. 3 (1972), 275.

[20] K. Ẑyczkowski and I. Bengtsson, Open Sys. Inf. Dyn. 11 (2004), 3.

[21] M. Horodecki, P. W. Shor, M. B. Ruskai, Rev. Math. Phys. 15 (2003), 629;

M. B. Ruskai, ibid. 15 (2003), 643.

[22] C. J. Zhang, Y. S. Zhang, S. Zhang, G. C. Guo, Phys. Rev. A 77 (2008), 060301(R).

[23] O. Gühne, P. Hyllus, O. Gittsovich and J. Eisert, Phys. Rev. Lett. 99 (2007), 130504. 